\title{
EFICIENCIA DE FUNGICIDAS EN EL CONTROL DE LA FLORA FÚNGICA TRANSPORTADA POR SEMILLAS DE ZANAHORIA (Daucus carota L.) Y SU RELACIÓN CON LA CALIDAD FISIOLÓGICA ${ }^{1}$
}

\begin{abstract}
RICARDO JOSE NOVO², ADRIANA VIGLIANCO ${ }^{3}$, MARIA ALEJANDRA PEREZ ${ }^{4}$, ALICIA CAVALLO ${ }^{5}$
ABSTRACT - Los objetivos de este trabajo fueron identificar los hongos asociados a las semillas de zanahoria de procedencia local y determinar la eficiencia del control químico sobre la flora fúngica transportada, en relación con la calidad fisiológica. Se analizó la calidad fisiológica y sanitaria de lotes de semillas de zanahoria provenientes de Córdoba, Argentina y se evaluó el efecto de tratamientos combinados de fungicidas en diferentes dosis y tiempos de inmersión. Se observó alta incidencia de hongos transportados con predominio de A. alternata, Fusarium sp. y Epicoccum sp los cuales afectaron la germinación y vigor de las semillas. Los tratamientos fungicidas redujeron el porcentaje de semillas infectadas y su efecto dependió principalmente del tiempo de inmersión. Los tratamientos más eficientes fueron Tebuconazole, Tiram y Carboxin + Tiram en dosis de 1.000 y 500 ppm de p.a. aplicados por inmersión durante una hora. Estos tratamientos indujeron además un incremento de la germinación de semillas de zanahoria.
\end{abstract}

Términos índices: control químico, calidad fisiológica, germinación, vigor, sanidad

\section{EFFICIENCY OF FUNGICIDES IN THE CONTROL OF SEEDBORNE FUNGI OF CARROT}

SEEDS (Daucus carota L.) AND PHYSIOLOGIC QUALITY OF SEEDS

\begin{abstract}
The objectives of this study were to identify the fungi associated to carrot seeds from Montecristo, Córdoba in Argentina and to determine the efficiency of the chemical seed treatment to control fungi and check the effect of this treatment on the physiological quality of the seeds. Seed health and physiological tests were carried out. The effect of fungicide combined treatments was evaluated considering the different doses and time of seed immersion. High incidence of associated fungi was observed with prevalence of Alternaria alternata, Fusarium sp. and Epicoccum sp. Seed germination and vigor levels were closely related to the levels of fungal incidence. The fungicidal treatments reduced the percentage of contaminated seeds and their effects depended mainly on the time of seed immersion. The most efficient treatments were obtained with Tebuconazole, Tiram and Carboxin+Tiram at doses of 1.000 and $500 \mathrm{ppm}$ a.i. applied by immersion for one hour. These treatments also induced increase in the germination percentages of carrot seeds under these study conditions.
\end{abstract}

Index terms: chemical control, physiological quality, germination, vigor, health

\footnotetext{
${ }^{1}$ Submetido em 17/10/2008. Aceito para publicação em 30/06/2009.

${ }^{2}$ Ing. Agr.(MSc.), Profesor Titular Departamento de Protección Vegetal FCA/UNC, C.C. 509, 5000 Córdoba Argentina.rjnovo@agro.uncor.edu.

${ }^{3}$ Biól., Auxiliar Departamento de Protección Vegetal FCA/UNC, C.C. 509, 5000 Córdoba Argentina. aviglian@agro.uncor.edu.
}

${ }^{4}$ Ing. Agr.(MSc.), Departamento de Biología Aplicada FCA/UNC, C.C. 509, 5000 Córdoba Argentina. maperez@agro.uncor.edu.

${ }^{5}$ Ing. Agr.(MSc.), Profesor Adjunto Departamento de Protección Vegetal FCA/UNC, C.C. 509, 5000 Córdoba Argentina. acavallo@agro.uncor.edu. 


\section{INTRODUCCION}

La producción de cultivos hortícolas es un importante rubro en la economía de la provincia de Córdoba, Argentina, y es una de las áreas de mayor extensión del denominado "Cinturón Verde" que rodea a la Ciudad. En el esquema de producción vigente, las semillas son un insumo en su mayoría importado. Sin embargo, se producen semillas de aquellos cultivares adaptados a la región, de menor rentabilidad y que demandan una baja inversión. Entre las especies multiplicadas con el fin de producir semillas se destacan las Umbelíferas, principalmente zanahoria y perejil. En la zona de producción mencionada, la primavera y el otoño son húmedos, en consecuencia existe una alta probabilidad de que la semilla esté asociada a hongos que disminuyan su germinación y vigor. En relación a ello, se desconoce la incidencia de los niveles de infección fúngica sobre la calidad fisiológica de semillas de zanahoria, en condiciones locales de producción.

Estudios realizados en Mendoza, Argentina (Makuch, 1979), evidenciaron la presencia de Alternaria porri fsp. dauci y A. radicina en semillas de zanahoria de distinto origen. En este estudio se destaca a estas especies como patógenos altamente transmitidos por semilla y en particular a $A$. radicina como el causante de apreciables pérdidas en cultivos destinados a la producción de semillas.

Así mismo los trabajos llevados a cabo por Tahvonen (1979); Soteros (1979); Cofelice y Porta-Puglia (1996); Coles y Wicks (2003) identificaron a Alternaria dauci, A. radicina y $A$. tenuis como patógenos asociados a las semillas de zanahoria. Por su parte, Souza et al. (2003) observaron baja incidencia en semillas tanto de Alternaria dauci como de Cercospora carotae. Sin embargo, Muñiz y Porto (1999) informaron la presencia de $A$. dauci asociado al embrión y endosperma, mientras que $A$. alternata y $A$. radicina prevalecieron en el pericarpio. En relación a la flora fúngica, Wahid et al. (1988) determinaron la presencia de A. radicina, A. alternata, Aspergillus flavus y Fusarium solani, entre otros. Así mismo, Hashmi et al. (1990) detectaron A. alternata, Cladosporium sp., Fusarium moniliforme, F. oxysporum, F. semitectum, Macrophomina phaseolina, Penicillium sp. y Aspergillus candidus. A su vez, Boughalleb et al. (2006) identificaron A. tenuísima, A. dauci, A. tenuis, Epicoccum sp y F. oxysporum. Mientras que Nowicki (1995); Tylkowska y Grabarkiewics-Szczesna (1995) señalaron la presencia de A. radicina y A. alternata como los hongos más importantes, determinándose también la presencia de Fusarium spp., Phoma spp. y Botrytis cinerea así como, la toxigenicidad de aislamientos de A. alternata para semillas y plántulas de zanahoria. Además en estudios realizados en semillas de zanahoria almacenadas por Kononkov y Dudina (1986), se citaron a Aspergillus glaucus, A. versicolor, A. candidus y una especie no identificada de Penicillium como los principales patógenos que aceleran el proceso de deterioro de las semillas durante el almacenamiento.

En relación al control de los hongos transportados por las semillas de zanahoria, Weingart y Stivers (1999) recomiendan el tratamiento de la semilla con Tiram previo a la siembra. Sin embargo, Tahvonen (1979), Utyugova y Levteeva (197); Soteros (1979); Galati y Mckay (1995) obtuvieron buenos resultados aplicando tratamientos de calor y Tiram. Cabe destacar que las dosis, los tiempos de exposición y las temperaturas empleadas fueron diversas en los distintos trabajos realizados. Contrariamente Standberg (1984) determinó bajo control de A. dauci en semillas tratadas con Tiram. Del mismo modo la aplicación de Tiram dio mejor resultado que los tratamientos con Iprodione solo o en mezcla con Metalaxil más Zineb (Rod, 1988).

Por otro lado, Cho et al. (1988) controlaron efectivamente A. dauci con Carboxin + Tiram, Benomil + Tiram o Captan, pero no controlaron A. alternata. Los estudios que vinculan los tratamientos fungicidas aplicados y la calidad fisiológica de la semilla son escasos. Al respecto, Mirkova (1981) determinó que Carboxin en mezcla con Tiram y Fuberidazole, fueron efectivos en el control de $A$. dauci y A. radicina y que las semillas tratadas mantuvieron su poder germinativo después de dieciocho meses de almacenamiento. Así mismo, Biniek y Tylkowska (1987) demostraron que semillas de zanahoria tratadas con Tiram por inmersión en acetona y acondicionadas con PEG 6000 germinaron mejor y en menos tiempo que las no tratadas. En tanto que el tratamiento con Tiram y PEG 8000 redujo la pérdida de germinación durante el almacenamiento comparado con las semillas sin tratar (Biniek, 2001).

Los objetivos de este trabajo fueron identificar los hongos asociados a las semillas de zanahoria procedente de la región de Córdoba, Argentina y determinar la eficiencia del control químico sobre la flora fúngica transportada y su relación con la calidad fisiológica.

\section{MATERIALES Y METODOS}

\section{a- Identificación de flora fúngica transportada en semillas de zanahoria.}

Se emplearon dos lotes (Lote 1 y 2 ) de semillas 
comerciales de zanahoria Daucus carota var. Royal Chantenay, procedentes de la localidad de Montecristo (latitud: $31^{\circ} 21^{\prime} \mathrm{S}$; longitud: $63^{\circ} 57^{\prime} \mathrm{O}$ ), provincia de Córdoba, Argentina. La determinación de la flora fúngica asociada a las semillas se llevó a cabo a través del método de "Blotter test modificado" (De Tempe y Binnerts, 1979; Mathur y Kongsdal, 2003).

Las semillas previamente desinfectadas de manera superficial con solución de hipoclorito de sodio al 1,5\% durante dos minutos y luego enjuagadas dos veces con agua estéril (Novo y Menezes, 1984); se colocaron sobre papel de filtro humedecido con agua destilada, en cápsulas de Petri tipo pirex desinfectadas con alcohol al 95\%. Se sembraron 50 semillas por caja, con cuatro repeticiones por lote.

Las semillas se incubaron durante 7 días en cámara a $20 \pm 2$ ${ }^{\circ} \mathrm{C}$ y ciclos de 12 horas de luz NUV y 12 horas de oscuridad (Neergaard, 1977).

Se determinaron los hongos asociados a la semilla con ayuda de claves (Barnett y Hunter, 1972; Malone y Musket, 1964; Ellis, 1971; Booth, 1971; Singh et al. 1992). La variable registrada fue el porcentaje de semillas infectadas con cada uno de los géneros encontrados.

\section{b- Determinación del efecto del nivel de infección sobre la germinación y el vigor de semillas de zanahoria.}

- Germinación: se sembraron entre papel 4 repeticiones de 100 semillas de zanahoria del lote 1 y 2 y se mantuvieron a $20-30{ }^{\circ} \mathrm{C}, 8$ horas luz y 16 horas de oscuridad (ISTA, 2003). A los 14 días se evaluó el porcentaje de plántulas normales anormales, semillas frescas y muertas.

- Vigor por crecimiento de plántulas: se sembraron 4 repeticiones de 40 semillas cada una de cada lote evaluado. La siembra se realizó entre papel humedecido con agua destilada en cámara oscura a $20 \pm 2{ }^{\circ} \mathrm{C}$ por un período de 14 días (ISTA, 1995). Al finalizar el ensayo se determinó el crecimiento de plántula $(\mathrm{cm})$ de acuerdo a lo propuesto por ISTA (1995).

\section{c - Determinación de la eficiencia de fungicidas aplicados a las semillas}

Se trabajó con semillas del lote 1 que presentaban alto nivel de infección, amplia diversidad de géneros presentes y mayor poder germinativo, de acuerdo a las evaluaciones previas.

Los fungicidas ensayados fueron: Carboxin + Tiram FS $20 \%+20 \%$ (5,6 dihidro-2-metil-1,4 oxatiin-3-carboxanilida mas bisulfuro de tetrametil tiocarbamilo), PCNB 75\% WP (pentacloronitrobenceno), Procimidone 50\% SC (N-(3,5diclorofenil)-1,2-dimetil ciclopropano-1,2-dicarboximida), Tebuconazole $25 \%$ EW, ( $\alpha-[2-(4$ clorofenil)etil]- $\alpha-(1-1$ dimetiletil)-1 H-1,2,4 triazol-1 etanol) y Tiram SC $20 \%$.

Se emplearon tres concentraciones de principio activo: 250, 500 y 1000 ppm y dos tiempos de aplicación: 1 minuto y 1 hora. Ésta se realizó por el método de inmersión; se sumergieron las semillas en cada concentración de fungicida durante los respectivos tiempos de tratamiento con posterior oreado sobre papel toalla en condiciones ambientales (temperatura ambiente de $20 \pm 5{ }^{\circ} \mathrm{C}$ y humedad relativa $65 \%$ \pm 10 ).

En el tratamiento testigo, las semillas fueron desinfectadas con hipoclorito de sodio al $1,5 \%$ durante dos minutos y luego lavadas dos veces con agua estéril (Novo y Menezes, 1984).

Cada tratamiento (combinación de fungicida, dosis y tiempo de aplicación), se evaluó por el método de Blotter test modificado (De Tempe y Binnerts, 1979). Se incubaron 4 repeticiones de 50 semillas cada una en cajas de Petri sobre papel de filtro humedecido con agua destilada, en cámara a $20 \pm 2{ }^{\circ} \mathrm{C}$ y ciclos de 12 horas de luz NUV y 12 horas de oscuridad (Neergaard , 1977).

A partir de los 7 días de incubación, y a medida que se desarrollaban las fructificaciones, se determinaron los hongos asociados a la semilla con las técnicas normalmente utilizadas en fitopatología y con la ayuda de claves (Barnett y Hunter, 1972; Malone y Musket, 1964; Ellis, 1971; Booth, 1971, Singh et al. 1992). Se registró el porcentaje de semillas infectadas y la frecuencia de los diferentes hongos en relación a los tratamientos aplicados.

El diseño fue totalmente aleatorizado y los resultados de las variables evaluadas fueron sometidos a análisis de varianza previa verificación de los supuestos sobre los términos. Para la comparación de las medias se utilizó test de Tukey con 5\% de probabilidad. El análisis de los datos se realizó con el paquete estadístico InfoStat (2001).

\section{RESULTADOS Y DISCUSIÓN}

De acuerdo a los resultados encontrados, la flora fúngica asociada a semillas de zanahoria presenta especies de hongos diferentes con diversa frecuencia de aparición (Tabla 1). En el lote 1 predominó Alternaria alternata, mientras que en el lote 2 fue más importante Fusarium sp. El predominio de $A$. alternata ya fue informada por Tahvonen (1979) y Cho et al. (1988) en semillas de zanahoria. Sin embargo, en los lotes evaluados no se determinó la presencia de Alternaria dauci 
mencionados por varios autores (Tahvonen, 1979; Utyugova y Levteeva, 1978; Mirkova, 1981; Standberg, 1884; Cho et al., 1988; Boughalleb et al., 2006). Tampoco se observó la presencia de Alternaria radicina citada en zanahoria por Tahvonen (1979), Utyugova y Levteeva (1978), Mirkova (1981), Cho et al. (1988); Cofelice y Porta-Puglia (1996); Coles y Wicks (2003).

TABLA 1. Flora fúngica asociada a semillas de zanahoria (Daucus carota L.) en diferentes lotes comerciales provenientes de Montecristo, Córdoba, Argentina.

\begin{tabular}{lcc}
\hline \multirow{2}{*}{ Especies de Hongos } & \multicolumn{2}{c}{ Lemillas infectadas $(\%)$} \\
\cline { 2 - 3 } Alternaria alternata & 66,3 & Lote 2 \\
Epicoccum sp & 6,3 & 33,3 \\
Fusarium sp & 0,0 & 50,3 \\
Rhizopus stolonifer & 5,4 & 8,6 \\
Aspergillus flavus & 5,1 & 0,3 \\
Aspergillus niger & 0,0 & 0,3 \\
Chaetomium globosum & 0,0 & 0,3 \\
Curvularia sp & 0,0 & 0,3 \\
Trichoderma sp & 4,7 & 2,7 \\
Drechslera sp & 2,1 & 0,3 \\
Penicillium sp & 0,0 & 3,7 \\
Phoma sp & 0,0 & 2,4 \\
\hline
\end{tabular}

En relación a la calidad fisiológica (Tabla 2) el lote 2, de mayor nivel de infección, presentó menor valor tanto de germinación como de vigor. Se observó mayor proporción de semillas muertas y entre las anormalidades se destacó la aparición de plántulas con raíz primaria podrida como resultado de infección por hongos. Estudios realizados por Tylkowska y Grabarkiewics-Szczesna (1995) señalaron la toxigenicidad de $A$. alternata lo que según estos autores, reduce tanto la viabilidad como el vigor en semillas y plántulas de zanahoria.
TABLA 2 Calidad fisiológica en términos de germinación y vigor de diferentes lotes de semillas de zanahoria (Daucus carota L.) provenientes de Montecristo, Córdoba Argentina.

\begin{tabular}{|c|c|c|c|}
\hline \multicolumn{2}{|l|}{ Ensayos } & Lote 1 & Lote 2 \\
\hline \multirow{7}{*}{$\begin{array}{c}\text { Germinación } \\
(\%)\end{array}$} & Plántulas normales & 58 & 38 \\
\hline & Plántulas anormales & 13 & 25 \\
\hline & Raíz $1^{\circ}$ atrofiada- & 2 & 0 \\
\hline & ausente & 7 & 20 \\
\hline & Raíz $1^{\circ}$ podrida & 4 & 5 \\
\hline & Hipocotilo podrido & 24 & 20 \\
\hline & $\begin{array}{l}\text { Semillas frescas } \\
\text { Semillas muertas }\end{array}$ & 5 & 17 \\
\hline Vigor & Aéreo & 5,17 & 4,72 \\
\hline $\begin{array}{c}\text { Crecimiento } \\
\text { de plántulas } \\
(\mathrm{cm})\end{array}$ & Radicular & 6,02 & 4,98 \\
\hline
\end{tabular}

La disminución de la calidad fisiológica de los lotes de semillas de zanahoria evaluados, indica que resulta necesario el tratamiento con fungicidas, a fin de mejorar la germinación y posterior crecimiento de plántulas. En relación a ello, los resultados de calidad obtenidos (Tabla 3), indican que todos los tratamientos fungicidas aplicados, en las tres dosis y dos tiempos de inmersión redujeron significativamente $(\mathrm{p}<0,05)$ el porcentaje de semillas de zanahoria infectadas con los distintos géneros. Tiram y Tebuconazole disminuyeron significativamente $(p<0,05)$ el porcentaje de semillas infectadas en todas las dosis ensayadas y tiempos de inmersión. El eficiente control de hongos patógenos con tratamientos con Tiram fue observado por Makuch (1979) y Standberg (1984) y la aplicación de este fungicida en caliente por Utyugova y Levteeva (1978) y Soteros (1979).

Por otra parte, en el tratamiento de Carboxin + Tiram, el nivel de control fúngico con inmersión por una hora fue significativamente superior $(\mathrm{p}<0,05)$ respecto a un minuto, en las 3 dosis ensayadas (Tabla 3). Resultados similares fueron obtenidos por Mirkova (1981) en el control de Alternaria. En general los tratamientos evaluados por otros autores (Mirkova, 1981; Standberg, 1984; Rod, 1988) fueron más eficientes a mayor concentración coincidiendo con los resultados de este trabajo. Por otra parte PCNB y Procimidone, si bien redujeron significativamente el porcentaje de semillas infectadas respecto al testigo, fueron los menos eficientes en todos sus tratamientos. 
TABLA 3. Porcentaje de semillas infectadas y germinación de semillas de zanahoria tratadas con diferentes fungicidas, dosis y tiempos de inmersión.

\begin{tabular}{|c|c|c|c|c|}
\hline \multicolumn{3}{|c|}{ Tratamientos } & $\begin{array}{c}\text { Semillas infectadas } \\
(\%)^{* * *}\end{array}$ & $\begin{array}{c}\text { Germinación } \\
(\%)^{* * * *}\end{array}$ \\
\hline Fungicidas & Tiempo* & Dosis** & & \\
\hline \multirow{6}{*}{ TIRAM } & \multirow{3}{*}{1} & $\mathrm{~A}$ & 16.0 cde & 63,7 bcdefg \\
\hline & & $\mathrm{B}$ & $17.5 \mathrm{de}$ & $58,2 \mathrm{ab}$ \\
\hline & & $\mathrm{C}$ & $22.5 \mathrm{e}$ & 62,0 abcde \\
\hline & \multirow{3}{*}{2} & $\mathrm{~A}$ & $5.0 \mathrm{ab}$ & 69,0 cdefgh \\
\hline & & $\mathrm{B}$ & 14.5 bcde & 63,2 bcdefg \\
\hline & & $\mathrm{C}$ & $22.5 \mathrm{e}$ & $59,0 \mathrm{ab}$ \\
\hline \multirow{6}{*}{ TEBUCONAZOLE } & \multirow{3}{*}{1} & $\mathrm{~A}$ & $5.0 \mathrm{ab}$ & $71,0 \mathrm{efgh}$ \\
\hline & & $\mathrm{B}$ & $6.0 \mathrm{abc}$ & 70,0 defgh \\
\hline & & $\mathrm{C}$ & $18.0 \mathrm{de}$ & 70,0 defgh \\
\hline & \multirow{3}{*}{2} & $\mathrm{~A}$ & $0.0 \mathrm{a}$ & $76,0 \mathrm{~h}$ \\
\hline & & $\mathrm{B}$ & $0.0 \mathrm{a}$ & $72,7 \mathrm{gh}$ \\
\hline & & $\mathrm{C}$ & $5.0 \mathrm{ab}$ & $72,0 \mathrm{fgh}$ \\
\hline \multirow{6}{*}{$\begin{array}{l}\text { CARBOXIN + } \\
\text { TIRAM }\end{array}$} & \multirow{3}{*}{1} & $\mathrm{~A}$ & $60 \mathrm{hi}$ & 64,7 bcdefg \\
\hline & & $\mathrm{B}$ & $60.0 \mathrm{hi}$ & 62,0 abcde \\
\hline & & $\mathrm{C}$ & 61.5 hif & $60,0 \mathrm{abc}$ \\
\hline & \multirow{3}{*}{2} & $\mathrm{~A}$ & $4.5 \mathrm{ab}$ & $76,0 \mathrm{~h}$ \\
\hline & & $\mathrm{B}$ & $5.0 \mathrm{ab}$ & $76,0 \mathrm{~h}$ \\
\hline & & $\mathrm{C}$ & $11.5 \mathrm{bcd}$ & 70,0 defgh \\
\hline \multirow{6}{*}{ PCNB } & \multirow{3}{*}{1} & $\mathrm{~A}$ & $68.0 \mathrm{ijk}$ & $59,5 \mathrm{abc}$ \\
\hline & & $\mathrm{B}$ & $71.0 \mathrm{jkl}$ & $53,0 \mathrm{a}$ \\
\hline & & $\mathrm{C}$ & 79.51 & $56,2 \mathrm{ab}$ \\
\hline & \multirow{3}{*}{2} & A & $24.5 \mathrm{e}$ & 64,2 bcdefg \\
\hline & & $\mathrm{B}$ & $37.5 \mathrm{f}$ & 65,7 bcdefg \\
\hline & & $\mathrm{C}$ & $54.0 \mathrm{gh}$ & 62,5 abcdef \\
\hline \multirow{6}{*}{ PROCIMIDONE } & \multirow{3}{*}{1} & $\mathrm{~A}$ & $59.5 \mathrm{hi}$ & 63,0 bcdef \\
\hline & & $\mathrm{B}$ & 63.5 hijk & $59,0 \mathrm{ab}$ \\
\hline & & $\mathrm{C}$ & $74.0 \mathrm{kl}$ & $56,5 \mathrm{ab}$ \\
\hline & \multirow{3}{*}{2} & $\mathrm{~A}$ & $47.0 \mathrm{fg}$ & 64,2 bcdefg \\
\hline & & $\mathrm{B}$ & $54.5 \mathrm{gh}$ & $60,0 a b c$ \\
\hline & & $\mathrm{C}$ & $63.0 \mathrm{hij}$ & $56,2 \mathrm{ab}$ \\
\hline \multirow[t]{2}{*}{ TESTIGO } & 1 & & $100.0 \mathrm{~m}$ & $61,0 \mathrm{abcd}$ \\
\hline & 2 & & $100.0 \mathrm{~m}$ & $58,0 \mathrm{ab}$ \\
\hline DMS & & & 10.9 & 9,5 \\
\hline
\end{tabular}

Tiempo* 1: un minuto 2: una hora

Dosis** A: 1000 ppm B: 500 ppm C: 250 ppm

*** medias seguidas de la misma letra no difieren significativamente por el test de Tukey $(\alpha=0,05)$

Respecto al control diferencial entre los géneros, Tebuconazole en ambos tiempos de inmersión y por Carboxin la incidencia de A. alternata disminuyó por acción del + Tiram y Tiram a la mayor dosis y tiempo de inmersión. 
Estos resultados son coincidentes a los encontrados en las evaluaciones de aplicación de Tiram mas calor (Galati y Mckay, 1995) y en mezcla con acetona (Biniek y Tylkowska, 1987); sin embargo se contraponen a lo que sostiene Cho et al (1988) en relación al control de Alternaria con Tiram solo y en mezcla.

Una especie no identificada de Fusarium ( $F$. avenaceum?) fue controlada por Tiram, Tebuconazole y Carboxin + Tiram en dosis alta y una hora de inmersión. Similares resultados se observaron en el control de Epicoccum sp. El control de Phoma sp fue muy eficiente con todos los fungicidas estudiados.

Respecto al efecto del tratamiento en relación a la calidad fisiológica, los fungicidas Tebuconazole y Carboxin + Tiram en sus dosis mayores y en el tiempo de aplicación de una hora lograron incrementar el poder germinativo de las semillas en aproximadamente $15 \%$. Los restantes fungicidas no difirieron significativamente $(\mathrm{p}<0,05)$ de los testigos, en relación a los valores de germinación observados. El efecto favorable producido por Tiram sobre la germinación, tasa de emergencia y crecimiento de plántulas ha sido observado por Muñiz y Porto (1999); Biniek y Tylkowska (1987); Magalhaes et al. (2004).

Al analizar el efecto de los funguicidas, independientemente de las dosis y tiempos de aplicación estudiados (Tabla 4), se observa que Tebuconazole fue el mas eficiente en la reducción del porcentaje de semillas infectadas, seguido por Tiram y Carboxin + Tiram. No se observó, en cambio diferencias significativas entre las dosis empleadas. En cuanto a los tiempos de aplicación, el nivel de respuesta en inmersión por una hora fue significativamente $(\mathrm{p}<0,05)$ superior a un minuto (Tabla $4)$. De lo que se deduce que el efecto del fungicida seleccionado para el mejor control del patógeno debe ser combinado con elevado tiempo de exposición siendo menor el efecto de la dosis. Estas condiciones no afectan la calidad fisiológica si no por el contrario mejoran el porcentaje de germinación, destacándose el Tebuconazole en dosis altas y mayor tiempo de inmersión (Tabla 4).

TABLA 4. Efecto del fungicida, dosis y tiempo de inmersión sobre el porcentaje de semillas infectadas y la germinación de semillas de zanahorias (Daucus carota $\mathrm{L}$.)

\begin{tabular}{|c|c|c|}
\hline Tratamientos & Semillas infectadas $(\%)^{*}$ & Germinación $(\%) *$ \\
\hline \multicolumn{3}{|l|}{ Fungicidas } \\
\hline Procimidone & $60.2 \mathrm{c}$ & $59,8 \mathrm{~b}$ \\
\hline PCNB & $35.7 \mathrm{~b}$ & $60,2 \mathrm{~b}$ \\
\hline Carboxin + tiram & $33.7 \mathrm{~b}$ & 68,2 a \\
\hline Tiram & $16.3 \mathrm{a}$ & $62,5 \mathrm{~b}$ \\
\hline Tebuconazole & $5.6 \mathrm{a}$ & $71,9 \mathrm{~b}$ \\
\hline DMS: & 14,4 & 3,9 \\
\hline \multicolumn{3}{|l|}{ Dosis } \\
\hline A: $1000 \mathrm{ppm}$ & 27,5 a & $67,1 \mathrm{a}$ \\
\hline $\mathrm{B}: 500 \mathrm{ppm}$ & 35,4 a & $64,0 \mathrm{ab}$ \\
\hline $\mathrm{C}: 250 \mathrm{ppm}$ & $41,4 \mathrm{a}$ & $62,4 \mathrm{~b}$ \\
\hline DMS: & NS & 3,6 \\
\hline \multicolumn{3}{|l|}{ Tiempos } \\
\hline 1: 1 minuto & $45.5 \mathrm{a}$ & $61,7 \mathrm{~b}$ \\
\hline 2: 1 hora & $23.2 \mathrm{~b}$ & 66,5 a \\
\hline DMS: & 9.0 & 2,2 \\
\hline
\end{tabular}

* medias seguidas de la misma letra no difieren significativamente por el test de Tukey $(\alpha=0,05)$

NS: no significativo

Se debe tener en cuenta que el incremento de la germinación no es tan alto como la reducción del porcentaje de semillas infectadas producido por los mismos fungicidas, lo que indica que, pese al control de los hongos, estos ya han 
causado daño a la semilla al momento de la evaluación.

\section{CONCLUSIONES}

Los lotes de semillas de zanahoria estudiados presentan alta incidencia de hongos transportados con predominio marcado de A. alternata y Fusarium sp. y en menor medida de hongos parásitos débiles como Epicoccum sp. y hongos de almacenamiento como Rhizopus stolonifer y Aspergillus flavus. Estos hongos asociados disminuyen la calidad fisiológica de las semillas en términos de viabilidad y vigor.

Los tratamientos fungicidas reducen el porcentaje de semillas infectadas y su efecto depende principalmente del tiempo de inmersión. Los tratamientos que presentan mayor eficiencia en el control de hongos trasportados por las semillas de zanahoria son Tebuconazole, Tiram y Carboxin + Tiram en dosis de 1.000 y 500 ppm de p.a. aplicado por inmersión durante una hora. Estos tratamientos inducen además un incremento de la germinación de semillas de zanahoria.

\section{REFERENCIAS}

BARNETT, H.L.; HUNTER, B.B. Ilustrated Genera of imperfect fungi. Burger Publishing Company. Minneapolis. 1972. 225p.

BINIEK, A.; TYLKOWSKA, K. Germination and mycoflore of carrot seeds treated with thiram and conditioned in polyethylene glycol (PEG 6000). Acta Horticulturae, v.215, p.411-416, 1987.

BINIEK, A. Influence of fungicide treatment and osmoconditioning with polyethylene glycol on germination of graded carrot seeds. Vegetable Crops Research Bulletin, v.55, p.115-120, 2001.

BOUGHALLEB, N.; TARCHOUN, N.; DALLAGI, W. Effect of fungicides on in vitro infestation level of Radish, Carrot and Pepper seeds. Plant Pathology Journal, v.5, n.3, p.388-392, 2006.

BOOTH, C. The Genus Fusarium. Commonwealth Micological Institute. England. 1971. 237p

CHO, S.H.; KANG, H.G.; KIM, C.H. Fungi associated with foreing seeds of twenty three plant species from six countries. Korean Journal of Plant Pathology, v4, n.4, p.319-324, 1988.

COFELICE, G.; PORTA-PUGLIA, A. Indagine sulla micoflora delle sementi di alcune plante ortensi. Informatore Fitopatologico, v.46,n12,p.21-25, 1996.
COLES, R.B. Y WICKS, T.J. The incidence of Alternaria radicina on carrot seeds, seedlings and roots in South Australia. Australasian Plant Pathology, v.32,n1,p.99-104, 2003.

DE TEMPE , J; BINNERTS , J. Introduction to methods of seed Health testing. Seed Science and Technology, v.7,p. 601-638, 1979.

ELLIS, M.B. Dematiaceous Hyphomicetes. Commonwealth Mycological Institute. England. 1971. 595p.

GALATI, A.; MCKAY, A.. Leaf blight diseases of carrots. Farmnote 73/95. Department of Agriculture, Western Australia. 2p. 1995.

HASHMI, R.; SHAUKAT,S.S.; KHANZADA, A.K.; MAHAMMAD, A. Efficacy of three detection techniques for the assessment of seed mycoflora of carrots in Sindh. Pakistan Journal of Botany, v.22, n.1, p.43-51, 1990.

INFOSTAT. Grupo InfoStat: version professional 1.1. Grupo InfoStat. Facultad de Ciencias Agropecuarias, Universidad Nacional de Córdoba, Argentina. Ed. Brujas Argentina S.A., 2001.

INTERNATIONAL SEED TESTING ASSOCIATTION. International Rules for seed testing. , Zürich: ISTA, 2003. $333 p$.

INTERNATIONAL SEED TESTING ASSOCIATTION. Handbook of vigor test methods. 3rd. Vigor Test Committee. Zürich: ISTA, 1995.

KONONKOV, P.F.; DUDINA, Z.N. Fungi on vegetable crop seed stored in condition of hight humidity and temperature. Seed Science and Technology, v.14, n.3, p.675-684, 1986.

MAGALHAES, F.H.L.; MACHADO, J. da C.; VIEIRA, M. das G.G.C.; GUIMARAES, R.M.; OLIVEIRA, J.A.; LEDO, C.A. da S. Desempenho de sementes de cenoura portadoras de especies de Alternaria apos o condicionamento fisiológico com adicão de tiram. Ciencia e Agrotecnologia, v.28, n.5, p.1007-1014, 2004.

MAKUCH, M.A. Reconocimiento y estudio de las enfermedades existentes en las semillas hortícolas. En: Resúmenes de la III Reunión Nacional de Olericultura. Mendoza, Argentina. 1979, p 21.

MALONE , L.P. y MUSKET , A.E. Seed borne Fungi . Description of 77 fungus species. Proceedings of International Seed Testing Association, v.29, p.176. 1964.

MATHUR, S.B. y KONGSDAL, O. Common laboraty seed health testng methods for detecting fungi. International Seed Testing Association, Zürich, p.89-314. 2003. 
MIRKOVA, E. Carrot seed treatment against fungal pathogens. Gradinarska Lozarska Nauka, Bulgaria, v.16,n.5/6, p.92-96, 1981.

MUÑIZ, M.F.B.; PORTO, M.D.M. Presença de Alternaria spp. em diferentes partes da semente de cenoura e em residuos culturais e efeito do tratamento de sementes na sua transmissão. Revista Brasileira de Sementes, v.21, n.1, p.187-193, 1999.

NEERGAARD, P. Seed Pathology. The Mac Millian Press Ltd. London, England, 1977. 877p.

NOVO, R.J.; MENEZES, M. Eficiencia de fungicidas no tratamento de sementes de sorgo granífero, Sorghum bicolor. Fitopatología Brasileira, v.9, p.543-549, 1984.

NOWICKI, B. Pathogenic mycoflora on carrot seeds. Acta Agrobotanica,v.48, n.2, p.49-57, 1995.

ROD, J. Treatment of vegetable seeds with fungicides. II Parsley, parsnips, carrots and celeriac. In: Seed Abstract, v.11, n.7, p.213, 1988.

SINGH, K.; FRISVAD, J.C.; THRANE, U.L.F.;MATHUR, S.B. An illustrated manual on identification of some seed borne Aspergilli, Fusaria, Penicillia and their mycotoxins. Denmark (Hellerup): Danish Government Institute of Seed Pathology for Developing Countries, p.133, 1992.

SOUZA, R.T., FORCELINI, C.A., REIS, E.M.; CALVETE, E.O. Freqüéncia de Alternaria dauci e Cercospora carotae como agentes da queima das folhas da cenoura em Passo
Fundo, RS. Fitopatologia Brasileira, v.26, n.3, p.614-618, 2003.

STANDBERG, J.O. Efficacy of fungicide against persistence of Alternaria dauci on carrot seeds Plant . v.68, n.1, p.39-42, 1984.

SOTEROS, J.J. Pathogenicity and control of Alternaria radicina and by $A$. dauci in carrots. New Zealand Jornal of Agricultural Research, v.22, n.1, p.191-196, 1979.

TAHVONEN, R. Seed borne fungi on Parsley and Carrot. Journal of the Scientific Agricultural Society of Finland, v.50, n.2, p.91-102, 1979.

TYLKOWSKA, K.; GRABARKIEWICZ-SZCZESNA, J. Toxigenicity of Alternaria alternata isolates from carrot seeds and seedlings. Seed Science and Technology, v.23, n.3, p.877-879,1995.

UTYUGOVA, T ; LEVTEEVA, N. Effectiveness of carrot seed disinfection against Alternaria disease. In: Review of Plant Pathology, v.57, n.3, p.139, 1978.

WAHID, A.; SHAUKUT, A.; SALEEM, A.. Seed borne mycoflora of carrot. Pakistan Journal of Agricultural Research, v.9, n.2, p.269-270, 1988.

WEINGART, D.; STIVERS, L. Crop profile for carrots in New York. Cornell Cooperative Extension, Ithaca, New York. 1999. Disponible en: www.ipmcenters.org/ cropprofiles/docs/ nycarrots.html. (08/02/2006). 